\title{
Immunomodulation on the ocular surface: a review
}

\author{
ANNA M. AMBROZIAK ${ }^{1,2}$, JERZY SZAFLIK ${ }^{1,3}$, JACEK P. SZAFLIK ${ }^{1,3}$, MACIEJ AMBROZIAK ${ }^{4}$, \\ JAN WITKIEWICZ ${ }^{5}$ PIOTR SKOPIŃSKI ${ }^{1,6}$ \\ ${ }^{1}$ SPKSO Ophthalmic University Hospital, Warsaw, Poland \\ ${ }^{2}$ Information Optics Department, Faculty of Physics, University of Warsaw, Poland \\ ${ }^{3}$ Department of Ophthalmology, Medical University of Warsaw, Poland \\ ${ }^{4}$ First Faculty of Medicine, Medical University of Warsaw, Poland \\ ${ }^{5}$ Ophthalmology Clinic “Twoje OKO”, Przemyśl, Poland \\ ${ }^{6}$ Department of Histology and Embryology Centre of Biostructure, Medical University of Warsaw, Poland
}

\begin{abstract}
The increasing understanding of immune mechanisms changed our perception of the ocular surface, which is now considered a compartment of the common mucosal immune system. It offered the possibility to alter the physiological immune response on the ocular surface and effectively combat inflammation, which impairs stability of the tear film and causes tear hyperosmolarity, causing symptoms of dry eye disease. The paper provides an overview of ocular surface anatomy and physiology, explains the underlying mechanisms of dry eye disease and discusses novel and promising treatment modalities, such as cyclosporine A, biological therapies using autologous serum and various growth factors as well as experimental treatment methods which are currently being investigated.
\end{abstract}

Key words: topical immunomodulation, ocular surface, topical cyclosporin, dry eye disease.

(Cent Eur J Immunol 2016; 41 (2): 195-208)

\section{Introduction}

Immunomodulation is defined as changing the physiological immune response, which can be either activated or suppressed using natural or pharmaceutical agents [1, 2]. It involves targeting specific inflammatory mediators to better control the disease and minimise the associated damage. The increasing understanding of immune mechanisms changed our perception of the ocular surface, which is now considered a compartment of the common mucosal immune system. It is also known that ocular tissues have low tolerance for inflammation, so even mild inflammation may cause vision impairment. Therefore, it is crucial to prevent the harmful effect of the excessive inflammatory response or chronic immune activation or revert it.

Among numerous topical medications, some available for years, which aim at tear substitution, tear preservation, stimulating tear production, and reducing or inhibiting inflammatory response, only few aim at restoring normal tear osmolarity and decreasing ocular surface damage in line with newer discoveries. The paper provides an overview of ocular surface anatomy and physiology, explains the underlying mechanisms of dry eye disease and discusses novel and promising treatment modalities, such as cyclosporine $\mathrm{A}$, biological therapies using autologous serum and various growth factors as well as experimental treatment methods which are currently being investigated.

\section{Anatomy and physiology of ocular surface}

The term "ocular surface" was introduced in 1977 by Thoft and Friend who emphasized the importance of the tear film as well as the connection between corneal and conjunctival epithelium in corneal reepithelialisation [3]. Recent studies show that the ocular surface not only shows functional properties but also capable of responding to external and internal stimuli. Actually, the tear film, cornea, palpebral and bulbar conjunctiva, main and accessory lacrimal glands, Meibomian glands, as well as eyelids and eyelashes with the associated glands, which constitute the structural and functional components of the ocular surface, show a characteristic immune profile [4-6]. It was also shown that the immunomodulation of this response is possible, which helps avoiding a potential detrimental effect of the excessive response or persistent immune activation [5-7]. It is essential as the two partly opposing roles of the immune protection include destroying the invading pathogens and limiting the inflammatory response, which may damage delicate ocular structures at the same time [8].

Correspondence: Anna M. Ambroziak, Department of Ophthalmology Medical University of Warsaw, Sierakowskiego 13, 03-709 Warsaw, Poland, e-mail: ambroziak.ann@gmail.com Submitted: 31.01.2016, Accepted: 29.02.2016 
Ocular surface and its immune system resemble other human mucous membranes in many aspects. Mucosal integrity can be maintained owing to the direct action of the lymphatic cells present in different forms within and closely underneath the epithelium or the secretion of soluble antibodies, which together form the 'mucosa-associated lymphoid tissue' (MALT) responsible for detecting antigens and triggering an immune response [9]. Thus, we can identify the conjunctiva-associated lymphoid tissue (CALT) within the conjunctiva, which constitutes a part of more extensive eye-associated lymphoid tissue (EALT). Like with any other MALTs, two forms of EALT can be distinguished: the 'organised' lymphoid tissue where lymphocytes are organized into lymphoid follicles, and the extensive 'diffuse' lymphoid tissue. The diffuse lymphoid tissue of CALT is mainly superficial, whereas the organised lymphoid tissue is situated deeper within the lamina propria and contains bone marrow-derived cells including lymphocytes, macrophages, granulocytes and mast cells. As the lymphoid cells form a thin, relatively erratic and unremarkable layer within the epithelium and connective tissue of the lamina propria, they had been considered inflammatory infiltrations until the mucosal component of the immune system became recognised. However, the projection of CALT onto the ocular surface showed that its topographical distribution is perfectly suitable to overlay the cornea when the eye is closed. Therefore, it provides effective immune protection to the cornea during blinking and overnight. Furthermore, it has an ability to detect corneal antigens and prime effector cells, as well as to distribute secretory $\operatorname{IgA}[10]$. On the other hand, the presence of the blood vessels ensures supplying nutrients and transporting metabolites as well as facilitates immune cell migration. This ensures that all innate and adaptive mechanisms work together in triggering and self-limiting an inflammatory response to an insulting factor [4, 5]. It should be noted, though, that ocular tissues are said to have low tolerance for inflammation. As a result, even mild inflammation may cause severe damage to ocular structures and significant vision impairment. It is due to the fact that inflammation causes chronic homeostatic imbalance within the lacrimal organ.

CALT contains all types of T cells. Cluster of differentiation (CD) is typically used for identifying molecules on the cell surface. The CD may work in different ways, being often either a receptor or a ligand. The two most known CD cells are CD4 (T-helper cells) and CD8 (cytotoxic T-cells). Conjunctival epithelium contains the most $\mathrm{CD}^{+}$ cytotoxic/suppressor T-cells. Among the lamina propria lymphocytes, the number of $\mathrm{CD} 4^{+} \mathrm{T}$-helper cells is either equal or slightly exceeds the number of $\mathrm{CD} 8^{+}$cells. $\mathrm{CD} 20^{+}$ B-cells (typical B-cell marker, binds to specific molecules present on many cells, including T-cells, stimulating the immune response) are also characteristic of lymphoid fol- licles. Diffuse conjunctival lymphocytes are mainly CD3 ${ }^{+}$ T-cells.

T-helper cells constitute a subpopulation of T-cells, which trigger the immune response. Over $90 \%$ of them are CD4 cell carriers. The CD4 cells recognise the antigens combined with the class II major histocompatibility complex cells (MHC). The antigen presenting cells (APC) within the lymph node trigger the activation of T-helper cells. It is followed by the release of cytokines, which play the immunoregulatory role. Based on the cytokine profile and their additional properties, the following subpopulations of T-helper cells have been distinguished:

- ThP cells, i.e. virgin T-helper cells, which express only interleukin (IL)-2,

- Th0 cells expressing IL-2, IL-3, IL-4, IL-5, IL-10, granulocyte-macrophage colony stimulating factor (GMCSF) and interferon $\gamma($ IFN- $\gamma$ ),

- Th1 cells expressing IL-2, IL-3, IFN- $\gamma$ and GM-CSF, are Th0-cell-derived, just like Th2 cells,

- Th2 cells expressing IL-4, IL-5, IL-10, IL-13 and GMCSF,

- Th3 cells expressing large amounts of transforming growth factor $\beta$ (TGF- $\beta$ ),

- ThM cells, Th memory cells, express only IL-2 and are activated more easily,

- Th17 cells, expressing mainly IL-17A and IL-17F, IL-21 and IL-22.

The basic structure of each immunoglobulin molecule includes two identical heavy chains and two identical light chains joined by disulfide bonds. There are different types of heavy chains (alpha, delta, gamma, mu and epsilon) and the type present in a molecule determines the immunoglobulin class (i.e., IgA, IgD, IgG, IgM and IgE) [11]. Immunoglobulins (Ig) produced by differentiated B cells are one of the basic elements of the secretory immune system in all types of human mucous membranes, including the conjunctiva. The most common conjunctival immunoglobulins are IgA polymers (p)IgA (the same antibody types prevail in the tear film as well). The vast majority of plasma cells within the lamina propria produce IgA, which has been confirmed by the immunohistochemical assay. They also produce low amounts of IgM. Other antibodies present in human conjunctiva are (p) $\operatorname{IgM}$ and trace amounts of $\mathrm{IgG}$.

Secretory IgA is an important, best-defined defence mechanism on the ocular surface. Specific IgA antibodies against the commensal conjunctival microbes are naturally produced. The presence of such pathogens as Acanthamoe$b a$ and Pseudomonas additionally triggers their expression. The IgA molecules bind to the microbes and immobilise them, ensuring their elimination as the tear film renews [12].

Mucins are large molecular weight, heavily glycosylated proteins with a protein core and a dense carbohydrate coating, which give the tear film its hydrophilic properties. As a result, the tear film is stable and the aqueous layer is 
spread evenly over the ocular surface $[13,14]$. They form glycocalyx, which, like scaffolding, ensures cell adhesion. Without this, tear film would not stay adherent to the ocular surface and damage could result [15]. The mucins present in the tear film maintain ocular surface hydration, provide lubrication and prevent friction of the ocular surface against the conjunctiva during the blink. Additionally, they support the epithelial barrier preventing microbial ocular damage.

Both corneal epithelium and conjunctival non-goblet cells express membrane-spanning mucins (such as MUC1, MUC2 and MUC4), while the conjunctival goblet cells produce secreted mucins (e.g. MUC5AC) [16]. Mucin production can also be induced by the inflammatory cytokines [e.g. IL-1 $\beta$, IL-6 and tumor necrosis factor $\alpha$ (TNF- $\alpha)$ ], as well as the stimulation of Toll-like receptors (TLR) in the corneal epithelium [14]. Additionally, conjunctival mast cells express multiple vasoactive mediators, such as histamine, heparin, cytokines (IL-4, IL-5, IL-6) and TNF- $\alpha$, which are essential to most inflammatory response mechanisms.

Collectively, the ocular mucosal tissues (cornea, corneal limbus, conjunctiva, conjunctival blood vessels, and eyelids), the tear secretory apparatus (main and accessory lacrimal glands, Meibomian glands, conjunctival goblet, and epithelial cells), and their innervation form an integrated, complex network referred to as the lacrimal functional unit (LFU) [17]. The input from the ocular surface tissues evokes the response, thus controlling the LFU via the neural pathway [18]. The corneal nerve endings send afferent impulses along the ophthalmic branch of the trigeminal nerve. This neurotransmission is integrated within the central nervous system and the paraspinal sympathetic tract and a response is generated in a form of efferent impulses stimulating secretion of the healthy tear film $[19,20]$. It maintains the homeostasis on the ocular surface, ensuring its integrity and essential for undisturbed function of the eye and the entire visual system [10].

\section{Dry eye disease}

Dry eye disease (DED) is a multifactorial disease of the tears and ocular surface which results in symptoms of discomfort, visual disturbance, and tear film instability with potential damage to the ocular surface. It is accompanied by increased osmolarity of the tear film and inflammation of the ocular surface. The term "dry eye" is considered a synonym of the term "keratoconjunctivitis sicca" (KCS) [21].

The two main types of dry eye are evaporative dry eye and hyposecretive (aqueous-deficient or tear-deficient) dry eye. Increased tear evaporation is involved in dry eye symptoms in $86 \%$ of patients. Just like tear deficiency, it is mainly a consequence of the disease/dysfunction affecting one or more components of the lacrimal functional unit
[20]. The classification of dry eye disease proposed by the International Dry Eye WorkShop (DEWS) is a comprehensive list of the said dysfunctions, which may be secondary to structural abnormalities, certain conditions, or lifestyle-related factors [21]. This etiopathogenic classification is presented in Table 1.

The underlying mechanisms leading to the development of DED are tear hyperosmolarity and instability of the tear film. The presence of one or both of them can be confirmed in a dry eye patient. The two mechanisms run in a vicious circle. The causes of initial events leading to localized autoimmunity can include environmental and/or microbial desiccating stress, which - if hormone imbalance and/or genetic predisposition are present - are sufficient to overcome the otherwise low ocular tissues' tolerance of inflammation leading to the dysfunction of the lacrimal functional unit (LFU), which alters tear composition and causes an imbalance to the ocular surface homeostasis, facilitating further tissue damage due to chronic inflammation.

The stress-induced increased tear film osmolarity causes hyperosmolarity of the ocular surface epithelial cells and acts as a pro-inflammatory stimulus to intensify the production and secretion of acute response cytokines, such as IL$1 \alpha$, IL-1 $\beta$, IL-6, IL-8 and TNF- $\alpha$, as well as matrix metal-

Table 1. Etiopathogenic classification of dry eye disease by the International Dry Eye WorkShop (DEWS) [21]

\begin{tabular}{l}
\hline Etiopathogenic classification of dry eye disease \\
\hline I. Aqueous tear-deficient dry eye \\
1. Sjögren's syndrome dry eye \\
2. Non-Sjögren's syndrome dry eye \\
$\frac{\text { 1) Primary lacrimal gland deficiencies }}{\text { 2) Secondary lacrimal gland deficiencies }}$ \\
$\frac{\text { 3) Obstruction of the lacrimal gland ducts }}{\text { 4) Reflex hyposecretion }}$ \\
\hline a) Reflex sensory block \\
\hline b) Reflex motor block \\
$\frac{\text { 1. Intrinsic causes }}{\text { 1) Meibomian gland dysfunction }}$ \\
$\frac{\text { 2) Disorders of lid aperture and lid/globe congruity }}{\text { or dynamics }}$ \\
$\frac{\text { 3) Low blink rate }}{\text { 3) Ocular surface disorders }}$ \\
\hline Extrinsic causes
\end{tabular}


loproteinases (MMP). The increased expression of MMPs - enzymes degrading the corneal epithelial basal cells and proteins, such as occludin, which ensure the corneal barrier integrity - further enhances the secretion of proinflammatory cytokines and chemokines and triggers the activation of corneal resident antigen presenting cells (APCs). The IFN- $\gamma$-induced squamous metaplasia, mucin alteration on corneal epithelial cells, the programmed death (apoptosis) of epithelial cells and goblet cells, as well as the IL-17-induced upregulation of MMP3/9 expression, which induces corneal epithelial barrier dysfunction, collectively contribute to tissue damage. After maturation, the APCs migrate to the regional lymph nodes, where they activate the Th1

Table 2. Meibomian gland conditions causing evaporative dry eye [21]

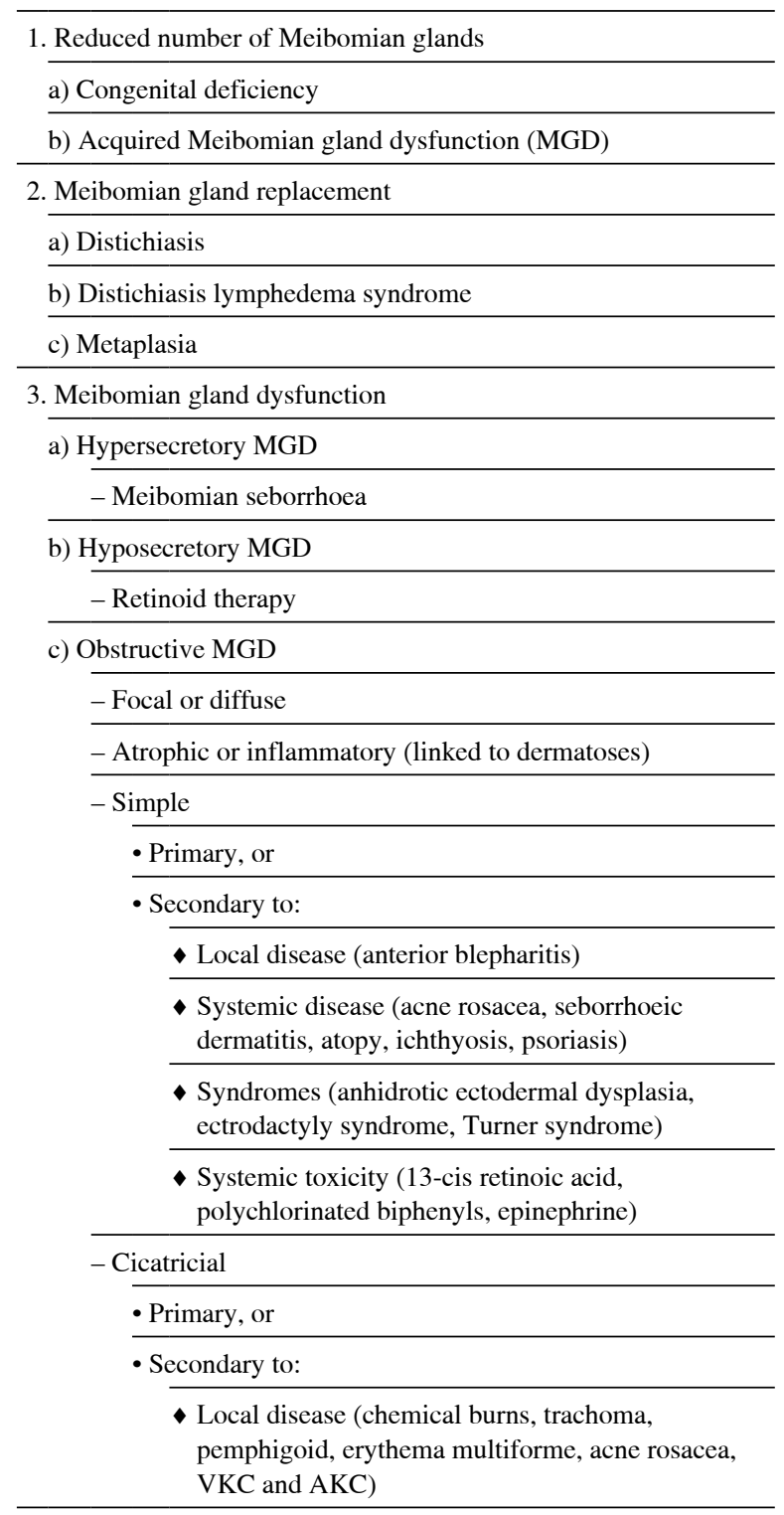

and Th17 cells. The activated $\mathrm{T}$ cells are attracted to the ocular surface tissues where they increase the chronic autoimmune activity. Furthermore, recent studies suggest that the secondary, complement-dependent tissue destruction is triggered by an autoantibody binding to antigens expressed in the lacrimal functional unit. On top of that, the lacrimal gland dysfunction exerts an indirect effect by decreasing the release of lactoferrin and other natural substances of known anti-inflammatory properties, inhibiting the natural protective mechanisms of the ocular surface [18].

Meibomian gland dysfunction (MGD) of sufficient extent and degree is associated with a deficient lipid layer of the tear film, increased tear evaporation, and as such it is considered one of the major causes of the evaporative dry eye [21]. The lipid layer of the tear film is produced by the Meibomian glands. Hence, their contribution to maintaining the stability and integrity of the tear film is essential. Abnormal composition of the tear film, including lipid deficiency or altered lipid composition in the external layer, results in a lack of adequate support to the ocular surface [18]. Therefore, Meibomian gland dysfunction (MGD) may be the implicated primary pathology, which subsequently causes the dry eye symptoms. The term Meibomian gland dysfunction was first used in a description of the condition in mid-1980s. Since that time, clinicians and researchers have coined and interchangeably used terms such as posterior blepharitis, Meibomian gland disease, meibomitis, meibomianitis, and Meibomian keratoconjunctivitis to refer to clinical conditions involving Meibomian gland and/or lid disease [22]. However, according to the International Workshop on Meibomian Gland Dysfunction, MGD is a chronic, diffuse abnormality of the Meibomian glands, commonly characterized by terminal duct obstruction and/or qualitativel quantitative changes in the glandular secretion. It may result in alteration of the tear film, symptoms of eye irritation, clinically apparent inflammation, and ocular surface disease [23].

The Meibomian gland conditions, which are implicated in pathogenesis of evaporative dry eye are shown in Table 2.

It is possible to quantify the severity of MGD using a grading system [24], as well as to assess the gland loss (meibography) [25] and the amount of oil in the lid margin reservoir (meibometry) [26]. All three investigations can provide directions during diagnosis and treatment monitoring.

Korb and Henriquez who studied a group of contact lens wearers with poor lens tolerance and mild symptoms of dry eye syndrome, were first to propose the hypothesis that Meibomian gland dysfunction, secondary to Meibomian gland orifice occlusion, primarily involves hyper-keratinization of ductal epithelium rather than the previously postulated inflammatory process within the gland [23, 27]. They observed the secretion within the dysfunctional Meibomian gland orifices, composed of desquamated epidermal cells and thick, waxy meibum. They also found that its 
manual expression and evacuation significantly improved contact lens wearing comfort and tear film stability. The histology studies in patients with severe dry eye syndrome and Meibomian orifices occluded with thick, viscous discharge confirmed the earlier findings. It was proved that hyper-keratinization of ductal epithelium was the main cause of cystoid dilatation of ductal orifice. It is also associated with squamous metaplasia of lymphoid follicles, which leads to the atrophy of structurally and functionally normal meibocytes as well as the cumulative reduction of the secreted meibum. No inflammatory cells were detected in the discussed pathologies [28].

As shown above, dry eye disease is a condition of multifactorial aetiology and nature, which universally combines chronic ocular surface inflammation with homeostatic imbalance it is secondary to, and - as such - can be environmentally modified. The following part of the article will discuss available treatment alternatives.

\section{Topical immunomodulatory drugs}

Topical immunomodulatory drugs (including immunosuppressants) exert their action by modulating (modifying or inhibiting) the immune response. It is a challenge as the dry eye disease shows a significant immune heterogeneity due to the fact that the number of known tear proteins is approximately 14000 .

\section{Cyclosporine A}

Cyclosporine A (CsA) an organic immunosuppressant, the first drug representative of a new generation of immunosuppressants. It is a neutral, lipophilic, cyclic undecapeptide composed of 11 amino acids. Its molecular weight is $1202.6 \mathrm{Da}$ and a molecular formula $\mathrm{C}_{62} \mathrm{H}_{111} \mathrm{~N}_{11} \mathrm{O}_{12}$. CsA was first isolated in 1971 from fungal species Tolypocladium inflatum as it turned out to be capable of inhibiting a mixed lymphocyte reaction (MLR) [29].

Very popular and well established in oncology and transplantology due to its capability of selective lymphocyte inhibition, topical Cyclosporine A was first used in ophthalmology in 1980, with the first report published in 1981 [30]. Following the initial approval of ophthalmic emulsion containing $0.5 \mathrm{mg} / \mathrm{ml}$ of cyclosporine A for increasing tear production in patients whose tear production is presumed to be suppressed due to ocular inflammation associated with keratoconjunctivitis sicca in 1983 [31], it was ultimately approved for ophthalmic indications by the U.S. Food and Drug Administration (FDA) in 2002. Cyclosporine $\mathrm{A}$ is used nowadays as an effective anti-inflammatory and anti-apoptotic agent. It is associated with a significantly reduced adverse effect rate as compared to conventional anti-inflammatory treatment with steroids.

The effect of cyclosporine involves inhibition of both cellular and humoral immune response, and modification of the inflammatory response. It does not affect lymphocyte migration, though. Cyclosporine prevents pathological apoptosis of secretory epithelium induced by the occlusion of non-specific pores in the mitochondrial membrane. These are responsible for the transient increase of membrane permeability for the molecules, which implies the increased tear film production [32-35]. Additionally, cyclosporine decreases expression of such cytokines as IL-2 (main mechanism), IL-3, IL-4, IL-5, TNF- $\alpha$, IFN- $\gamma$, as well as the T-cell growth factor (TCGF). It also affects T-helper cell activation. Inhibited IL-2 secretion in T-helper cells reduces and inhibits the CD4+ T-cell dependent hyperplasia thus limiting the activity of natural killer (NK) cells. As a result, the antibody production and macrophage activation decrease. Furthermore, cyclosporine shows a mild inhibitory effect on B-cells by inhibiting the induction phase of lymphoid cell proliferation. The effect of cyclosporine is reversible. It is not myelotoxic and it does not inhibit hemopoiesis. Being a selective immunosuppressant, it does not impair the phagocytic activity and migratory capacity of the reticuloendothelial system [30, 32-34].

Cyclosporine has a dual mechanism of action. Stage one involves cyclosporine binding to cyclophilin, its intracellular receptor and analogue of immunophilin, which is a T-cell cytoplasmic protein. Cyclophilin disrupts the molecular process within the T-cells, after their activation by the antigen presenting cells (APCs). Next, stage two involves binding of the cyclosporine-cyclophilin complex to the calcium-dependent serine-threonine phosphatase, known as calcineurin and its inactivation. This prevents dephosphorylation of the nuclear factor of activated $\mathrm{T}$ cells (NFAT) and its activation. As a result, IL-2 transcription is not initiated and IL-2 is not released. Acting early by blocking the resting lymphocytes in the G0 and G1 stages of a cell cycle, cyclosporine leads to their programmed cell death (apoptosis) [36].

Topical cyclosporine is effective in management of numerous eye conditions, including dry eye syndrome (Table 3). It is also used to prevent graft rejections in patients after keratoplasty [37]. The results of several studies support the use of cyclosporine in patients with MGD, rosacea and dry eye syndrome [33, 34]. Perry et al. showed a significant improvement involving decreased lid margin hyperaemia, telangiectasia and corneal staining, as well as the increased number of active glands in patients with MGD after 3-month use of topical cyclosporine [38,39]. The full list of indications for use of topical cyclosporine is presented in Table 3. According to the Summary of Product Characteristics, the $0.05 \%$ emulsion of cyclosporine $\mathrm{A}$ is indicated for low tear production due to ocular inflammation associated with keratoconjunctivitis sicca. The anti-inflammatory treatment regimen in severe dry eye disease includes CsA administration for 6 months twice daily. At the same time, the patient should use preservative-free tear substitutes. However, it is crucial to allow a 15-min- 
Table 3. The indications for local cyclosporine use [37]

\begin{tabular}{|c|c|}
\hline Clinical situations & Indications \\
\hline \multirow{13}{*}{$\begin{array}{l}\text { Selected ocular } \\
\text { pathologies }\end{array}$} & Meibomian gland dysfunction \\
\hline & Dry eye disease, esp. severe Sjögren's syndrome dry eye \\
\hline & Vernal keratoconjunctivitis (to improve pain and local inflammation) \\
\hline & Thygeson's superficial punctate keratopathy \\
\hline & Atopic keratoconjunctivitis \\
\hline & Epidemic adenoviral keratoconjunctivitis (to reduce the number of subepithelial deposits and recurrence rate) \\
\hline & Corneal ulcer (to reduce inflammation and improve healing) \\
\hline & Mooren's ulcer \\
\hline & Herpes simplex keratitis \\
\hline & Neurotrophic keratopathy \\
\hline & Anterior uveitis, esp. not responding to corticosteroids \\
\hline & Sympathetic ophthalmia \\
\hline & Autoimmune uveitis NOS \\
\hline \multirow{5}{*}{$\begin{array}{l}\text { Postoperative } \\
\text { conditions }\end{array}$} & After cataract surgery (to minimise local inflammation in patients with primary and secondary dry eye syndrome) \\
\hline & After pterygium surgery (to reduce the recurrence) \\
\hline & After glaucoma surgery \\
\hline & After corneal transplant (to prevent graft rejection, in high-risk grafts) \\
\hline & After refractive surgery (to improve corneal sensation - nerve fibre repair) \\
\hline
\end{tabular}

ute interval between the doses of the two preparations. An off-label treatment regimen assumes CsA administration up to 4 times daily [40].

Until recently (except for compound medications developed in sterile laboratory conditions), CsA was only available as $0.05 \%$ ophthalmic emulsion. It is still the most popular CsA formulation, but its properties limit its use - it is not effective within the posterior segment due to rapid elimination, it does not achieve therapeutic concentration within the cornea and ciliary body either [39, 40]. High lipophilicity of already used cyclosporine formulations hinders its topical administration. Patient quality of life and compliance are crucial for the long-term treatment effect. Therefore, the duration of treatment with cyclosporine emulsion must be relatively long, to be able to assess its effect. However, it is possible to use cationic nanoemulsions as vehicles for the active ingredients of topical medications in ophthalmology. This innovative technology has been developed and successfully launched over the last decade. Recently, a preservative-free cationic nanoemulsion of cyclosporine $(1 \mathrm{mg} / \mathrm{ml})$ has been approved by EMA for treatment of severe keratitis in adult patients with dry eye disease, which has not improved despite treatment with tear substitutes. This formulation contains a higher concentration of cyclosporine as compared to currently available $0.05 \%$ formulation. It shows an identical immunosuppression mechanism by inhibiting calcineurin, which reduces inflammation secondary to dry eye disease. The drug named Ikervis ${ }^{\circledR}$ (Sant- en Oy) is already available in some European countries. Its launch in Poland is planned for 2016.

The main challenge in development of this new formulation involved selecting a safe cationic vehicle (only anionic ones had been used until then), which could remain on the ocular surface for long enough. Pharmacological characteristics of the active substance constitute the critical factor, which determines the properties of the final medicinal product, e.g. its polarity or lipophilicity. On the other hand, the epithelial surface of the cornea is naturally hydrophobic; it acquires some hydrophilic properties owing to the normal, uncompromised structure of glycocalyx and mucin layer. The new cationic nanoemulsion is the "oil-inwater" solution based on Novasorb ${ }^{\circledR}$, the patent-protected cationic emulsion technology platform, which gives the drug its hydrophilic properties [39, 41].

Preclinical studies confirmed lack of toxicity, overall good tolerance and safety of the new formula. They also confirmed the positive effect of nanoemulsion on the ocular surface, even before the active substance was added. However, there is only minimum risk of ocular penetration, circulatory absorption and systemic effect of cyclosporine with topical administration to the conjunctival sac. Therefore, the formulation has a good safety profile in long-term treatment.

The SANSIKA study was a multicentre, randomised, double-masked, two parallel arm, vehicle-controlled, 6-month phase III trial a 6-month open-label treatment 
safety follow-up period to assess the efficacy and safety of Ikervis $^{\circledR}$ (CsA $1 \mathrm{mg} / \mathrm{ml}$ eye drops) administered once daily in adult dry eye patients with severe keratitis. It was conducted in 246 subjects, at 66 sites in 9 European countries.

The inclusion criteria were:

- Grade 4 corneal fluorescein staining (CFS), as measured on a modified Oxford scale,

- OSDI score of 23 or more at the screening and baseline visits,

- Schirmer's test without anaesthetic score of 2-10 mm/5 min at the screening visit.

After a wash-out period of 1-2 weeks, the subjects were randomised to receive Ikervis ${ }^{\circledR}$ or vehicle daily at bedtime for 6 months. Patients randomised to the vehicle group were switched to Ikervis ${ }^{\circledR}$ after 6 months. The primary endpoint was a confirmed response to treatment, defined as (a) the proportion of patients achieving at least a two-grade improvement in keratitis (CFS), and (b) a 30\% symptomatic improvement assessed using OSDI score by month 6. They were measured at the end of treatment in month 6 , before the switch and the beginning of a 6-month open-label treatment safety follow-up period [36].

The proportion of responders in the CsA group was higher as compared to the vehicle group (28.6\% vs. $23.1 \%$, respectively). However, the difference was not statistically significant. A statistically significant improvement in OSDI scores at month 6 as compared to baseline was shown in both groups. However, the difference between the two groups was not statistically significant. Corneal staining assessment showed significantly less staining in the CsA group as compared to the vehicle group at month 3 and 6 ( $p=0.024$ and $p=0.037$, respectively). Score conversion into the modified Oxford scale yielded the result of 1.50 , which means that, at month 6 , the patients in the vehicle group have on average $50 \%$ more punctate corneal lesions compared with the CsA group [42].

SICCANOVE study was the 6-month, multicentre, double-masked, vehicle controlled, supportive clinical trial in 492 dry eye patients with moderate to severe keratitis (CFS score of 2 to 4 ). The inclusion criteria included:

- Tear break-up time (TBUT) below $8 \mathrm{~s}$,

- Corneal fluorescein staining score ranging from 2 to 4 ,

- Schirmer's test (without anaesthetic): score of 2-10 $\mathrm{mm} / 5 \mathrm{~min}$,

- Lissamine green staining (LGS) grade 4 or more (van Bijsterveld),

- At least one sign/ symptom of DES (grade 2 or more, on a scale of 0 to 4 ).

The subjects were randomised to receive Ikervis or vehicle once daily, at bedtime, for a period of 6 months. The primary endpoints measured at the end of treatment in month 6 were (a) the change in CFS score and (b) the change in the global score of all symptoms of ocular discomfort unrelated to study medication (assessed using a Visual Analogic Scale). The observed difference in CFS improvement at month 6 between the two study groups was small but statistically significant in favour of Ikervis ${ }^{\circledR}$ (mean change from baseline in CFS was -1.05 and -0.82 with Ikervis ${ }^{\circledR}$ and vehicle, respectively; $p=0.009$ ). The mean change from baseline in ocular discomfort score was -12.82 and -11.21 with Ikervis ${ }^{\circledR}$ and vehicle, respectively $(p=0.808)[41]$.

Both discussed studies did not confirm a statistically significant improvement of symptoms in subjects receiving Ikervis ${ }^{\circledR}$ as compared to those receiving the vehicle after 6 months of treatment, regardless of a measuring scale (VAS or OSDI). However, the subgroup analysis in both studies showed that the subjects with Sjögren's syndrome (approximately one third of all enrolled in each study) reported a statistically significant improvement in CFS in favour of Ikervis ${ }^{\circledR}$ [36].

The new, sustained release cyclosporine formulas are being studied at the moment, with contact lenses, punctal plugs or subconjunctival implants proposed as vehicles [43]. The subconjunctival implant continuously releases cyclosporine for 12 months. It is recommended for use in patients after keratoplasty with the high risk of graft rejection and considered to be effective. However, the relatively high cost and the risk of adverse effects limit its use.

Owing to changed lipophilicity, a slower elimination rate and high tissue penetration, novel pharmaceutical formulations, such as the discussed Ikervis ${ }^{\circledR}$, will likely become the standard of ocular immunosuppression and immunomodulation.

\section{Other immunophilin binding drugs}

Like cyclosporine, tacrolimus and sirolimus block calcineurin and inhibit the activity of mature cells, not interfering with the cell maturation process.

Tacrolimus is a macrolide, which inhibits the production of IL-2 promoting the development and proliferation of $\mathrm{T}$ cells. By preventing calcium-dependent T-cell activation, it alters the cellular immune response. Therefore, it is used as an immunosuppressant. Currently, its oral and injectable formulations are FDA-approved and indicated for the prophylaxis of organ rejection in patients receiving allogeneic liver, kidney, or heart transplants [44].

A prospective double-blind randomized study of $0.03 \%$ tacrolimus eye drops administered twice daily for treatment of dry eye was conducted in 24 patients with dry eye related to Sjögren's syndrome. The patients were randomized to receive tacrolimus $(n=14)$ or vehicle (olive oil; $n=10$ ). The primary endpoint was the quantity of the tear film assessed using Schirmer's test. The secondary endpoints were the quality of the tear film (assessed with TBUT) as well as integrity of the ocular surface (assessed with corneal staining). All endpoints were ultimately assessed after 90 days of treatment and the follow-up as- 
sessments were scheduled for days 7, 14, 28 and 90 of treatment.

The $0.03 \%$ tacrolimus eye drops improved the tear film stability and ocular surface condition in patients with Sjögren's syndrome with the onset of improvement observed as early as on day 7 in fluorescein and Rose Bengal staining. This score continued to improve even more after 90 days. The mean Schirmer's and TBUT scores showed an improvement after 28 days of treatment as compared to baseline. There was no statistically significant improvement in corneal staining Schirmer's test and TBUT in the vehicle group [45].

Sirolimus, originally known as rapamycin, is a broad acting compound of well-established anti-inflammatory, immunosuppressive and antiproliferative properties. It is used for improving coronary luminal diameter in patients with symptomatic ischemic heart disease and in renal transplant patients as an immunosuppressive agent.

The phase II dose-ranging clinical study to assess the safety and efficacy of subconjunctival injection of sirolimus was conducted in a controlled adverse environmental (CAE) model in 143 patients with dry eye. The subjects were randomised to receive sirolimus at a dose of 220, 440 and 880 micrograms or placebo (controls) for 28 days. The primary endpoints were the mean fluorescein staining within the inferior corneal portion after CAE exposure and the mean ocular discomfort during CAE exposure. The secondary endpoints were treatment safety across treatment groups, and improvement demonstrated in additional evaluations of dry eye such as fluorescein and lissamine green staining, conjunctival redness, tear film break-up time, blink rate, ocular protection index, Schirmer's test, and corneal sensitivity [46]. The results confirmed an excellent safety and tolerance profile of sirolimus with no systemic adverse events noted, and demonstrated its bioactivity as an immunomodulatory agent, which decreased the need to use steroids for symptomatic improvement in study subjects [47].

\section{Biological therapy}

Other available treatment modalities include mainly biological therapy, that is:

- autologous serum eye drops [23],

- epidermal growth factor (EGF),

- TGF- $\beta$,

- substance P,

- $\beta 2$-macroglobulin,

- nerve growth factor (NGF),

- lymphocyte function-associated antigen 1 (LFA-1), and - anakinra.

Whereas biological therapy is effective and particularly indicated in corneal epitheliopathies, it should be noted that its clinical effect is likely to deteriorate after the treatment is discontinued, especially in patients with superficial corneal epitheliopathy. This treatment has not been
FDA- or EMA-approved yet. The next sections provide an overview of the most promising biological therapies to be used in dry eye patients.

\section{Autologous serum eye drops}

A composition of natural tears including water, salts, hydrocarbons, proteins, and lipids is too complex to be substituted with any man-made product [48]. Furthermore, in order to prevent microbial contamination, tear substitutes often contain chemical preservatives, which induce toxic and allergic reactions, especially in individuals with sensitive eyes [49]. That is why, after an initial attempt to use autologous serum eye drops (AS) in a continuous infusion for dry eye [50], Fox et al. attempted to formulate an effective, unpreserved tear substitute unavailable commercially at that time by diluting patient's autologous serum (AS) with saline solution and published the first report on the successful use of biological treatment in 1984 [51]. However, its use was really popularised by Tsubota, who described the effect of a 4-week treatment with $20 \%$ autologous serum-based eye drops on ocular surface re-epithelialisation in patients with severe dry eye secondary to Sjögren's syndrome, attributing this effect to high content of growth factors and vitamins [52]. Other reported treatments using blood-derived formulations included various AS concentrations between $20 \%$ and $100 \%$ with treatment efficacy seemingly dose-dependent, as well as platelet rich plasma (PRP) in gel, drops or suspension and plasma rich in growth factor (PRGF), all used for treating ocular surface disorders and their sequelae [53].

Serum is a liquid blood fraction, which remains after clotting. Hence, it does not contain red blood cells, blood platelets, fibrinogen and coagulation factors. However, it still contains growth factors (EGF, TGFB, FGB and IGF), as well as vitamin A, immunoglobulins, P-substance, lysozyme, lactoferrin, $\beta 2$-macroglobulin and lipids, which have the ability to restore the defects of the lipid fraction of the tear film in patients with severe dry eye disease secondary to Meibomian gland dysfunction or graft versus host disease (GvHD). The $\mathrm{pH}$ and osmolarity of autologous serum match these of natural tears and so does its composition, with the exception of lower immunoglobulin A (IgA), epithelial growth factor (EGF) and vitamin C content and higher vitamin A, lysozyme, TGF- $\beta$ and fibronectin content in serum than in tears [54]. Since many of the essential tear components are present in blood serum, it seems reasonable to use serum as a tear substitute, especially that the biochemical factors present in autologous serum, such as vitamin A, EGF, TGF- $\beta$, insulin-like growth factor 1 (IGF-1), nerve growth factor (NGF), fibronectin, substance $\mathrm{P}$ and other cytokines, are actively involved in proliferation, differentiation, and maturation of healthy epithelial cells. Therefore, a proposed mechanism of action of autologous serum as a tear substitute involves ocular surface lubrication and substituting biochemical tear 
components in order to maintain the stability of corneal and conjunctival epithelium [55]. It is applied as an unpreserved, autologous product, which lacks antigenicity and shows very limited toxicity. AS is an attractive treatment modality owing to numerous advantages such as excellent tolerance and lack of secondary preservative-induced damage [56]. The $20 \%$ autologous serum-based eye drops were shown to improve the tear break-up time (TBUT) by over 2 seconds, to enhance tear production, improve corneal staining in superficial corneal epitheliopathy and to reduce epithelial neoplasia [55]. The limitations of this approach include its "home-made" preparation process and lack of standardized preparation protocol as well as the presence of cytokines in its composition, which may adversely affect ocular tissue regeneration [57].

The blood for AS eye drops is donated during a single phlebotomy procedure performed under aseptic conditions. A typical blood donation yields approx. $100 \mathrm{ml}$ of blood (30-35 $\mathrm{ml}$ of serum), which in turn (after $20 \%$ dilution) provides the estimated three-month treatment batch (with a dosage scheme of 6-8 times daily), so the actual amount of blood to be donated is determined based on the expected duration of treatment. Normally, 100-200 ml of whole blood is drawn into sterile containers. However, it is also possible to collect larger volumes, up to $470 \mathrm{ml}$, using a sterile blood pack without anticoagulant. The containers are left for $2 \mathrm{~h}$ at room temperature to clot before they are centrifuged at 3,000 $\mathrm{g}$ for $15 \mathrm{~min}$ so as to separate serum from other morphotic elements. The supernatant (i.e. serum) is then aspirated using sterile 50-ml disposable syringes in a laminar airflow hood, which ensures sterile conditions. The collected volume is diluted $1: 5$ with sterile BSS. The obtained solution should be homogenised by gently shaking the vial and aliquoted through a $0.2-\mathrm{mm}$ filter into sterile $2 \mathrm{ml}$ dropper bottles. According to Fox, filtration removes fibrin strands, which are said to reduce the effect of serum eye drops. Serum eye drops should be stored frozen; thawed eye drops can be used up to 8 times daily and should be disposed of at the end of the day on which they were used $[58,59]$.

\section{Anakinra}

Anakinra (Kineret ${ }^{\circledast}$, Amgen Inc.) is a recombined unglycosylated antagonist of human interleukin-1 receptor (IL-1Ra) that targets the IL-1/IL-1R1 signal pathway to increase tear secretion and normalize mucin layer. A single molecule of anakinra, composed of 153 amino acids, is produced as a result DNA recombination using E coli gene expression system and its molecular weight is 17.3 $\mathrm{kDa}$. It is currently FDA-approved in an injectable form as either monotherapy or a part of polytherapy, for moderate to severe rheumatoid arthritis in patients, who have failed to respond to treatment with other disease modifying an-

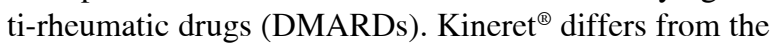

native human IL-1Ra by having an additional single methionine at the N-terminus [60].

Anti-inflammatory drugs successfully used in dry eye syndrome decrease IL-1 production and increase the formation of IL-1 receptor antagonists (IL-1Ra) on the ocular surface. IL-1Ra reduces the IL-1-dependent inflammation by blocking the binding of IL-1a and IL-1b to the IL-1 receptor. Topical use of IL-1Ra has turned out to be successful as experimental therapy of corneal graft rejection, dry eye syndrome, allergic conjunctivitis and ocular inflammation secondary to chemical burns in animals.

The prospective phase $1 / 2$, randomized, double-masked, vehicle-controlled clinical trial in seventy-five subjects with refractory dry eye disease (DED) associated with Meibomian gland dysfunction (MGD) with a 16-week follow-up showed that compared to the eye lubricant, $2.5 \%$ topical anakinra was four times more likely to bilaterally resolve corneal staining, which is an objective clinical measure of ocular surface disease. It was assessed to be six times more effective in reducing dry eye symptoms as compared to the ocular lubricant, which alone can improve the signs of DED to some extent. The study subjects, randomised in the $2: 2: 1$ ratio to receive artificial tears, $2.5 \%$ topical anakinra or $5 \%$ topical anakinra three times daily, reported the studied drug to be well tolerated and significantly more effective in improving the signs and symptoms of DED than the comparator. Furthermore, after anakinra was discontinued at week 12 , a trend was observed in both groups toward increased dry eye symptoms between weeks 12 and 16. This effect was not shown in patients receiving artificial tears, which confirms the therapeutic effect of anakinra [61].

\section{Off-label and experimental therapies}

Isunakinra (EBI-005) is a novel IL-1 receptor antagonist and the first protein specifically designed for topical use in ophthalmology for the treatment of ocular surface disorders, such as dry eye disease and allergic conjunctivitis (AC). Being the recombinant protein, isunakinra binds to the IL-1 receptor. It prevents both IL-1 subtypes (IL-1 $\alpha$ and IL-1 $\beta$ ) from binding to the IL-1 receptor and its signalling pathway is blocked. As a result, the most common symptoms of ocular surface diseases, such as discomfort, itching and inflammation, are resolved or significantly reduced.

The efficacy and safety of isunakinra in dry eye patients were evaluated in OASIS study (A Multi-Center, Double-Masked, Randomized, Controlled, Efficacy and Safety Study of EBI-005 $5 \mathrm{mg} / \mathrm{ml}$ Topical Ophthalmic Solution Versus Vehicle Control In Subjects With Moderate to Severe Dry Eye Disease). The primary endpoints were the mean change in corneal fluorescein staining score and the mean change in patient-reported improvement in pain and discomfort from baseline to week 12 . The safety and tolerability of isunakinra as compared to vehicle-control were also evaluated. 
The study showed no statistically significant difference in primary and secondary endpoints between the treatment group and the vehicle control group. The symptomatic improvement from baseline reported by patients in both groups was statistically significant. Actually, the change from baseline in corneal fluorescein staining score and patient-reported improvement of pain and discomfort was greater in controls than in the active treatment arm. However, the differences between the groups were not statistically significant. Isunakinra was well tolerated in Phase 3 (which confirms the findings of Phase 1/2 studies). There were no treatment-related serious adverse events and ocular irritation was reported by less than $5 \%$ of patients. Approximately $13 \%$ of patients in the study reported use of artificial tears, with no statistically significant difference in this respect between the active treatment group and the control group. It was concluded that the Phase 3 study in dry eye disease did not meet its primary efficacy endpoints. Therefore, the development of isunakinra for the use in dry eye disease and the previously planned second Phase 3 study were cancelled for now [62].

Various cytokines and recombinant growth factors have been proposed as tear substitutes to facilitate corneal re-epithelialisation, which is naturally mediated by epidermal growth factor (EGF), platelet derived growth factor (PDGF), and fibroblast growth factor (FGF). It was shown that the combination of these and other factors offers an improved, synergistic therapeutic effect. However, the major disadvantage of recombinant growth factor-based therapy is its high cost. It seems more reasonable and feasible for the payer to use patient's own blood-derived products to facilitate ocular tissue regeneration. Plasma rich in growth factors (PRGF) is autologous platelet rich plasma, which arises as a novel interesting alternative for the treatment of corneal pathologies. Rich in platelets and leukocyte-free, PRGF contains booster amounts of EGF, PDGF and FGF, which determine its biological and antimicrobial properties. Recent studies present PRGF as a promising approach for the treatment of several ocular surface diseases including dry eye, obtaining successful results even in patients who did not respond to autologous serum eye drops [57].

Experimental studies of epidermal growth factor (EGF) in a murine dry eye model induced by benzalkonium chloride (BAC) showed that topical application of EGF stabilized the tear film, increased corneal epithelial cell count and enhanced cell migration preventing apoptosis. As a result, it was effective in maintaining the integrity of corneal epithelium. These findings suggests potential of EGF as a therapeutic agent in clinical treatment of dry eye [63].

TGF- $\beta$ is a pleiotropic cytokine which - depending on context - can exert a pro-inflammatory or anti-inflammatory effect. Its level in patients with Sjögren's syndrome, one of most common causes of dry eye, was shown to be elevated. The study in an experimental murine model of dry eye induced by desiccating stress demonstrated symptomatic improvement after disruption of the TGF- $\beta$ signalling pathway. This suggests therapeutic potential of TGF- $\beta$ antagonists in dry eye disease [64].

Nerve growth factor (NGF) is a polypeptide discovered in early 1950 s by R. Levi Montalcini. NGF is an endogenous protein essential for sensory neuron survival and growth, as well as for CNS neuron differentiation. At the end of 1990s, the reports on the key role of NGF on the pathophysiology of the ocular surface were published, emphasizing its involvement in immunomodulation and trophic support on the ocular surface as well as conjunctival and corneal healing. NGF receptors are actually present within the cornea, conjunctiva, iris, ciliary body and lens. Their activation is crucial for differentiation of corneal epithelial cells, which implies the key role of NGF in pathophysiology of numerous anterior segment conditions, such as neurotrophic keratitis or dry eye syndrome $[65,66]$. As a result, the murine NGF (mNGF) eye drops were evaluated for efficacy and safety both in animal experimental models and in human patients with neurotrophic keratitis and corneal ulceration secondary to autoimmune inflammation [67]. Murine NGF (mNGF), harvested from submandibular glands of mice, is similar to human NGF in chemical structure. The amino acid sequence of murine NGF is identical in $90 \%$ with this of human NGF. Pre-clinical studies in animal models showed efficacy of murine NGF in improving corneal innervation after LASIK and increasing tear production in dry eye syndrome [68].

The newest treatment is the recombined human nerve growth factor (rhNGF) harvested from E. coli. It has the same biochemical structure as mNGF and pre-clinical studies confirmed that its activity was identical to mNGF.

Currently, a randomized, double-blind study (REPA$\mathrm{RO}$ ) is being conducted at 39 sites in nine European countries to assess the efficacy and safety of rhNGF-based eye drops $(10 \mu \mathrm{g} / \mathrm{ml}$ or $20 \mu \mathrm{g} / \mathrm{ml})$ administered 6 times daily to over 170 patients with moderate or severe (grades 2 and 3) neurotrophic keratitis secondary to diabetes, herpetic ocular infections, neurosurgical interventions and other related diseases. Along with the efficacy and safety primary endpoints, the secondary endpoints include the evaluation of corneal lesion healing, improvement in visual acuity and in corneal sensitivity [69].

Cis-urocanic acid (cis-UCA) is an endogenous small molecule of the skin showing local anti-inflammatory and anti-proliferative properties. It has a proven strong cytoprotective effect on the ocular epithelial cells where it suppresses inflammatory response and reverses the UVB stress-induced loss of cell viability. Preclinical studies in rabbit models of ocular inflammation demonstrated a strong potential of the cis-UCA ophthalmic solution in the treatment of inflammatory eye conditions, since an inflammation, just as any chronic stress is associated with epithelial cell cytotoxicity [70]. 
The Phase 1 study to assess the tolerability and safety of the patent-protected cis-UCA ophthalmic formulation as well as its pharmacokinetics after single and repeated dosing was conducted in the EU in 37 healthy volunteers. They were randomised to receive either $0.5 \%$ or $2.5 \%$ cis-UCA eye drops, or placebo. The study was conducted in two parts: the first one involved eye drop administration to one eye, three times daily on a single day, whereas the second one was a continuation of previous treatment with eye drop administration to both eyes, three times daily for 14 days [71].

The results confirmed the safety as well as excellent local and systemic tolerance of cis-UCA eye drops throughout the study. There were no differences in ocular safety parameters between cis-UCA and placebo. In terms of ocular comfort, subjects receiving cis-UCA reported burning of the eyes statistically more often than their counterparts receiving placebo. However, this reaction was transient and mild in all cases. The study confirmed potential having detectable (below $10 \mu \mathrm{g} / \mathrm{ml}$ ) urine cis-UCA concentrations. However, its plasma concentrations were insignificant and negligible [72].

The phase 2, multicentre, randomized, double-masked, placebo-controlled clinical study to assess the safety and efficacy of $1.0 \%$ and $2.5 \%$ cis-UCA ophthalmic solutions was performed in 161 subjects with dry eye, in line with the proposed indication, that is treatment of ocular surface inflammation typically seen in the dry eye syndrome. The primary endpoints, assessed at month 1 , were corneal fluorescein staining and symptom score. The inclusion criteria were:

- age of 18 years or more,

- informed consent,

- previous history of dry eye,

- having used eye drops in the past or wishing to use them.

However, contact lens wearers, patients after LASIK, those treated with cyclosporine within the last month, as well as pregnant and breastfeeding women were excluded [73].

None of the two cis-UCA solutions showed a statistically significant improvement in the two primary endpoints, as compared to placebo. However the results suggest that cis-UCA eye drops are safe and as well tolerated as placebo while they showed a significant benefit compared to placebo in some secondary endpoints [74].

Lifitegrast 5.0\% ophthalmic solution (Shire Pharmaceuticals) is a topical, novel small-molecule integrin antagonist and IL-1 receptor blocker. It exerts a therapeutic effect via inhibition of lymphocyte functional antigen 1 (LFA-1) and precluding its binding to intercellular adhesion molecule-1 (ICAM-1). As the interaction between the two discussed molecules does not take place, T-cells are not activated, so the inflammatory cytokines are not released, which reduces T-cell-mediated inflammation [75].

OPUS-3 was a phase 3 efficacy and safety study of lifitegrast versus placebo in patients with dry eye disease. Subjects were randomised to receive lifitegrast or placebo twice daily for 84 days (12 weeks). The inclusion criteria were:

- a recent (less than 1 month) history of artificial tear use, and

- an eye dryness score (EDS) $\geq 40$.

The results of OPUS-3 replicated the primary patient-reported endpoint of OPUS-2, a phase 3 efficacy and safety study $(p<0.0001)$. OPUS- 3 met the primary endpoint of significantly improved Eye Dryness Score from baseline to day 84 (treatment difference of 7.16 [95\% CI], $3.04,11.28 ; p=0.0007)$. Additionally, OPUS-3 met the secondary endpoints of improved Eye Dryness Score from baseline to days 14 and 42 (treatment difference (95\% CI) of $7.85(4.33,11.37)$ and $9.32(5.44,13.20)$ respectively; $p<0.0001$ for both endpoints) [76].

\section{Other treatment modalities}

Anti-inflammatory treatment is becoming increasingly more popular as the first-line treatment in ocular surface disorder, especially in the context of inflammatory aetiology of the condition and the role of immune processes in its clinical course.

A typical treatment regimen involves lubricants and topical steroids used four times daily for up to one month and twice daily (tapered dose) for two consecutive months. One of the most common topical steroids is loteprednol etabonate $0.2 \%$ or $0.5 \%$, which - like cyclosporine - is indicated for long-term treatment with twice daily administration.

Although considered effective, this treatment modality is associated with several disadvantages, such as a relatively high cost, potential risk of steroid-induced systemic adverse effects including IOP spikes, impaired healing, decreased immune response or local adverse effects, for instance stinging sensation induced by cyclosporine. It should also be noted that cyclosporine is approved for dry eye, whereas steroids, although included in numerous treatment algorithms, are still used off-label in ocular surface disorder. Non-steroid anti-inflammatory drugs are not recommended as they interfere with re-epithelialisation.

When addressing the immunomodulatory treatment, the authors deliberately referred only briefly to the wide range of possibilities available with steroid anti-inflammatory drugs. For more information, please see our previous review articles [40, 77, 78].

An interesting, novel approach to treating corneal epithelial defects was postulated by Altýnörs et al. [79] who suggested using human breast milk to enhance corneal healing. Their study in mice showed that human breast milk accelerates corneal re-epithelialisation more efficiently than autologous serum or tear substitutes. However, such treatment appears to be a bit too controversial.

Discussing immunomodulatory treatment, current diagnostic trends in ocular surface disease, including bio- 
marker analysis, should also be mentioned. We are still in a constant search of a minimally invasive parameter, useful in diagnosis and treatment of dry eye, additionally contributing to our better understanding of the underlying immune mechanisms. Since ocular surface constitutes a part of the mucosal immune system, epithelial lymphocytes are proposed as potential biomarkers of abnormalities associated with the most common ocular surface disorders, such as Meibomian gland dysfunction and dry eye disease. Inflammatory markers (IL-1, IL-6, IL-8, class II MHC, TNF- $\alpha$, APC, and HLADR, whose level was shown to increase four-fold in patients with ocular surface disease) appear to be the best candidates [80].

Therefore, the most interesting proposed assays are epithelial expression of HLA-DR+ molecules, which detects upregulation related to the increased immune activity of epithelial cells, and tear film cytokine analysis. Secretory phospholipase A2 Type IIA (sPLA2-IIa) is the most frequently mentioned potential trigger of the inflammatory process within the conjunctiva and cornea of all tear film cytokines [81]. Other clinically evaluated biomarkers include such proinflammatory cytokines as IL-17, IL-23, IL-1b, MUC1 and MUC4. They all make excellent markers - but of the disease rather than of its treatment.

The commercially available tests known for their high sensitivity, specificity and reproducible results are e.g. InflammaDry Detector using the Rapid Pathogen Screening (RPS) technology in order to quantify the level of extracellular matrix metalloproteinase 9 (MMP-9) in the tear film.

\section{Conclusions}

Dry eye syndrome shows immune heterogeneity (there are approximately 14,000 known tear proteins). Therefore, an individualised treatment approach is required. The possibility to use modern, nanotechnology-based pharmacological approaches adds a significant amount to the existing treatment alternatives.

The authors declare no conflict of interest.

The authors gratefully acknowledge the assistance of Karolina Kalisz, BA (www.medical-interpreter.pl) in drafting and editing the manuscript.

\section{References}

1. Radomska-Leśniewska DM, Skopiński P, Bałan BJ, et al. (2015): Angiomodulatory properties of Rhodiola spp. and other natural antioxidants. Centr Eur J Immunol 40: 249-262.

2. Rokicki D, Zdanowski R, Lewicki S, et al. (2014): Inhibition of proliferation, migration and invasiveness of endothelial murine cells culture induced by resveratrol. Centr Eur J Immunol 39: 449-454.
3. Thoft RA, Friend J (1977): Biochemical transformation of regenerating ocular surface epithelium. Invest Ophthalmol Vis Sci 16: 14-20.

4. Ambroziak AM, Krawczyk P, Skopiński P (2013): Zaburzenia powierzchni oka w ujęciu immunologicznym ze szczególnym uwzględnieniem spojówki i nabłonka rogówki. Kontaktologia i Optyka Okulistyczna 1: 16-19.

5. Skopiński P, Krawczyk P, Ambroziak AM (2013): Immunology of the ocular surface and contact lens wear: theoretical fundamentals. Centr Eur J Immunol 38: 254-259.

6. Ambroziak AM, Krawczyk P, Szulborski K, Skopiński P (2013): Clinical implications of the immunity of the ocular surface: the effect of extended contact lens wear. Centr Eur J Immunol 38: 260-264.

7. Barabino S, Chen Y, Chauhan S, Dana R (2012): Ocular Surface Immunity: Homeostatic Mechanisms and Their Disruption in Dry Eye Disease. Progress in Retinal and Eye Research 31: 271-285.

8. Knop E, Knop N (2007): Anatomy and immunology of the ocular surface. Chem Immunol Allergy 92: 36-49.

9. Knop E, Knop N (2000): Conjunctiva-associated lymphoid tissue in the human eye. Invest. Ophthalmol Vis Sci 41: 12701279.

10. Knop E, Knop N (2005): The role of eye-associated lymphoid tissue in corneal immune protection. J Anatom 206: 271-285.

11. Seamone C, Jackson WB (2013): Immunology of the External Eye. In: Tasman W, Jagger EA (eds.). Duane's Clinical Ophthalmology. Vol. 4 Ch. 2, Lippincott-Raven, Philadelphia.

12. Knop E, Knop N, Claus P (2008): Local production of secretory $\operatorname{IgA}$ in the eye-associated lymphoid tissue (EALT) of the normal human ocular surface. Invest Ophthalmol Vis Sci 49: 2322-2329.

13. Gibson IK, Kunert KS, Argüeso P (2000): Regulation of mucin gene expression in the ocular surface epithelia. Cornea 19: 97.

14. Rolando M, Zierhut M (2001): The ocular surface and tear film and their dysfunction in dry eye disease. Surv Ophthalmol 45: 203-210.

15. Watanabe H (2002): Significance of mucin on the ocular surface. Cornea 21: 17-22.

16. Dartt DA, Dana R, D'amore P, et al. (2011): Immunology, inflammation and diseases of the eye. Elsevier, Groton.

17. Stern ME, Beuerman RW, Fox RI, et al. (1998): The pathology of dry eye: the interaction between the ocular surface and lacrimal glands. Cornea 17: 584-589.

18. Stern ME, Schaumburg CS, Pflugfelder SC (2013): Dry Eye as a mucosal autoimmune disease. Int Rev Immunol 32: 19-41.

19. Beuerman RW, et al. (2004): The Lacrimal Functional Unit in Dry Eye and Ocular Surface Disorders. Pflugfelder SC, Beuerman RW, Stern ME (eds.). Marchel Dekker, New York, 11-39.

20. Lemp MA, et al. (2012): Distribution of aqueous-deficient and evaporative dry eye in a clinic-based patient cohort: a retrospective study. Cornea 31: 472-478.

21. Report of the International Dry Eye WorkShop (DEWS) (2007). Ocul Surf 5: 65-204.

22. Nichols K (2011): The International Workshop on Meibomian Gland Dysfunction: Introduction. IOVS 52: 1917-1921.

23. Nichols KK, Foulks GN, Bron AJ, et al. (2011): The International Workshop on Meibomian Gland Dysfunction: Executive Summary. Invest Ophthalmol Vis Sci 30; 52: 1922-1929. 
24. Bron AJ, Benjamin L, Snibson GR (1991): Meibomian gland disease. Classification and grading of lid changes. Eye 5: 395 411.

25. Mathers W, Shields W, Sachdev M, et al. (1991): Meibomian gland dysfunction in chronic blepharitis. Cornea 10: 277-285.

26. Yokoi N, Mossa F, Tiffany JM, Bron AJ (1999): Assessment of meibomian gland function in dry eye using meibometry. Arch Ophthalmol 117: 723-729.

27. Geerling G, Tauber J, Baudouin C, et al. (2011): The international workshop on meibomian gland dysfunction: report of the subcommittee on management and treatment of meibomian gland dysfunction. Invest Ophthalmol Vis Sci 2011; 52: 2050-2064.

28. Korb DR, Henriquez AS (1980): Meibomian gland dysfunction and contact lens intolerance. J Am Optom Assoc 51: 243-251.

29. Survase SA, Kagliwal LD, Annapure US, Singhal RS (2011): Cyclosporin A - a review on fermentative production, downstream processing and pharmacological applications. Biotechnology Advances 29: 418-435.

30. Hunter PA, Wilhelmus KR, Rice NSC, et al. (1981): Cyclosporin A applied topically to the recipient eye inhibits corneal graft rejection Clin Exp Immunol 45: 173-177.

31. Summary of Product Characteristics. Restasis ${ }^{\circledR}$ ophthalmic emulsion $0.5 \mathrm{mg} / \mathrm{mL}$ (2012), Allergan.

32. Daull P, Lallemand F, Philips B, et al. (2011): Distribution of cyclosporin A in ocular tissues after topical administration of cyclosporin A-cationic emulsion to pigmented rabbits. IOVS; 186: 376.

33. Kaswan RL (1988): Intraocular penetration of topically applied cyclosporin. Transplantation Proceedings 20 (suppl.): 650-655.

34. Lallemand F, Felt-Baeyens O, Besseghir K, et al. (2003): Cyclosporin A delivery to the eye: a pharmaceutical challenge. Eur J Pharmaceutics Biopharmaceutics 56: 307-318.

35. Yokoi N (2013): Dry eye syndrome: basic and clinical perspectives. Future Medicine Ltd, UK, doi: 10.2217/9781780842646.

36. Summary of Product Characteristics. Ikervis ${ }^{\circledR} 1 \mathrm{mg} / \mathrm{ml}$ eye drops, emulsion. (2015) Santen UK Limited.

37. Stevenson W, Chauhan SK, Dana R (2012): Dry eye disease: an immune-mediated ocular surface disorder. Arch Ophthalmol 130: 90-100.

38. Perry HD, Doshi-Carnevale S, Donnenfeld ED, et al. (2006): Efficacy of commercially available topical cyclosporin A $0.05 \%$ in the treatment of meibomian gland dysfunction. Cornea 25: 171-175.

39. Perry HD, Doshi S, Donnenfeld ED, et al. (2003): Double Masked Randomized Controlled Study Evaluating Topical $0.05 \%$ Cyclosporin A in the Treatment of Meibomian Gland Dysfunction (Posterior Blepharitis). IOVS; 44: 1395.

40. Lallemand F, Daull Ph, Benita S, et al. (2012): Successfully Improving Ocular Drug Delivery Usingthe Cationic Nanoemulsion, Novasorb. J Drug Delivery 2012: 604204.

41. Buggage RR, Amrane M, Ismail D, et al. (2011): The effect of Cyclokat (unpreserved $0.1 \%$ cyclosporin cationic emulsion) on corneal involvement in patients with moderate to severe dry eye disease participating in a phase III, multicenter, randomized, controlled, double-masked, clinical trial. Eur J Ophthalmol doi: 10.5301/EJO.2011.7544.

42. Leonardi A (2015): Ikervis® $1 \mathrm{mg} / \mathrm{ml}$ cyclosporin - a new therapeutic option for the treatment of severe keratitis in dry eye disease. Changing paradigms of treating severe keratitis in dry eye disease - ocular surface symposium report. Proceed- ings of a Symposium Presented at the European Society of Ophthalmology 2015 Congress in Vienna, Austria on 7 June.

43. Gupta C, Chauhan A (2011): Ophthalmic delivery of cyclosporin A by punctal plugs. J Controlled Release 150: 70-76.

44. Summary of Product Characteristics. Prograf®: tacrolimus capsules, tacrolimus injection for intravenous infusion only (2009). Astellas Pharma US, Inc.

45. Moscovici BK, Holzchuh R, Sakassegawa-Naves FE, et al. (2015): Treatment of Sjögren's syndrome dry eye using $0.03 \%$ tacrolimus eye drop: Prospective double-blind randomized study. Contact Lens and Anterior Eye 38: 373-378.

46. NCT00814944 Study Record Detail (2016). Available at: clinicaltrials.gov (Access: 2016, January 23).

47. Colligris B, Crooke A, Huete-Toral F, Pintor J (2014): An update on dry eye disease molecular treatment: advances in drug pipelines. Expert Opinion on Pharmacotherapy 15: 13711375.

48. Dogru M, Tsubota K (2011): Pharmacotherapy of dry eye. Expert Opin. Pharmacother 12: 325-334.

49. Quinto GG, Campos M, Behrens A (2008): Autologous serum for ocular surface diseases. Arq Bras Oftalmol 71 (6 Suppl): 47-54.

50. Ralph RA, Doane MG, Dohlman CH (1975): Clinical experience with a mobile ocular perfusion pump. Arch Ophthalmol 93: 1039-1043.

51. Fox RI, Chan R, Michelson JB, et al. (1984): Beneficial effect of artificial tears made with autologous serum in patients with keratoconjunctivitis sicca. Arthritis Rheum 27: 459-461.

52. Tsubota K, Goto E, Shimmura S, et al. (1999) Treatment of persistent corneal epithelial defect by autologous serum application. Ophthalmology 106: 1984-1989.

53. Management and therapy of dry eye disease: report of the Management and Therapy Subcommittee of the International Dry Eye WorkShop (2007). 2007; 5: 163-178.

54. Bradley JC, Bradley RH, McCartney DL, Mannis MJ (2008): Serum growth factor analysis in dry eye syndrome. Clin Experiment Ophthalmol 36: 717-720.

55. Pan Q, Angelina A, Zambrano A, et al. (2013): Autologous serum eye drops for dry eye. Cochrane database 8: CD009327; doi:10.1002/14651858.CD009327.pub2.

56. Geerling G, MacLennan S, Hartwig D (2004): Autologous serum eye drops for ocular surface disorders. Br J Ophthalmol 88: 1467-1474.

57. Anitua E, de la Fuente M, Muruzabal F, et al. (2015): Plasma rich in growth factors (PRGF) eye drops stimulates scarless regeneration compared to autologous serum in the ocular surface stromal fibroblasts. Experimental Eye Research 135: 118-126.

58. Wróbel-Dudzińska D, Pyszniak E, Kuna A, et al. (2014): Produkty krwiopochodne w leczeniu zespołu suchego oka. Okulistyka (2014): 69-71.

59. Geerling G, Hartwig D (2006): Autologous serum eyedrops for ocular surface disorders. In: Reinhard T, Larkin DFP (eds.). Essentials in Ophthalmology: Cornea and External Eye Disease. Springer Berlin Heidelberg New York, 1-20.

60. Summary of Product Characteristics. Kineret ${ }^{\circledR}$ (anakinra) (2006). Amgen Inc.

61. Amparo F, Dastjerdi MH, Okanobo A, et al. (2013): Topical interleukin 1 receptor antagonist for treatment of dry eye disease: a randomized clinical trial. JAMA Ophthalmol 131: 715-723.

62. Media release dated May 18, (2015): Eleven Biotherapeutics, Inc. 
63. Xiao X, He H, Lin Z, et al. (2012): Therapeutic effects of epidermal growth factor on benzalkonium chloride-induced dry eye in a mouse model. Invest Ophthalmol Vis Sci 53: 191-197.

64. De Paiva CS, Volpe EA, Gandhi NB, et al. (2011): Disruption of TGF- $\beta$ signaling improves ocular surface epithelial disease in experimental autoimmune keratoconjunctivitis sicca. PLoS ONE 6: e29017. doi: 10.1371/journal.pone.0029017.

65. Lambiase A, Mantelli F, Sacchetti M, et al. (2011): Clinical applications of NGF in ocular diseases. Arch Ital Biol 149: 283-292.

66. Bonini S, Aloe L, Bonini S, et al. (2002): Nerve growth factor (NGF): an important molecule for trophism and healing of the ocular surface. Adv Exp Med Biol 506 (Pt A): 531-537.

67. Ferrari MP, Mantelli F, Sacchetti M, et al. (2014): Safety and pharmacokinetics of escalating doses of human recombinant nerve growth factor eye drops in a double-masked, randomized clinical trial. BioDrugs 28: 275-283.

68. Ma K, Yan N, Huang Y, et al. (2014): Effects of nerve growth factor on nerve regeneration after corneal nerve damage. Int J Clin Experimental Med 7: 4584-4589.

69. Sacchetti M, Lambiase A (2014): Diagnosis and management of neurotrophic keratitis. Clinical Ophthalmology 8: 571-579.

70. Viiri J, Jauhonen HM, Kauppinen A, et al. (2009): Cis-urocanic acid suppresses UV-B-induced interleukin- 6 and -8 secretion and cytotoxicity in human corneal and conjunctival epithelial cells in vitro. Molecular Vision 15: 1799-1805.

71. Jauhonen HM, Kari E, Pylkkänen L, et al. (2015): A randomized phase I clinical study of cis-urocanic acid eye drops in healthy adult subjects. Acta Ophthalmol doi: 10.1111/ aos.12651.

72. Leino L, Jauhonen H, Kari E, et al. (2012): Cis-urocanic acid eye drops are safe and well tolerated in healthy adults - results from a randomised phase 1 clinical study. Acta Ophthalmologica 90; doi: 10.1111/j.1755-3768.2012.T104.x.

73. NCT02326090 Study Record Detail, clinicaltrials.gov, accessed on January 23 (2016).

74. Herantis Pharma Plc. Company media release dated 3 June (2015), accessed on January 23, 2016.

75. Tauber J, Karpecki P, Latkany R, et al. (2015): Lifitegrast ophthalmic solution $5.0 \%$ versus placebo for treatment of dry eye disease. Ophthalmology 122: 2423-2431.

76. Shire plc. Company media release dated October 27 (2015), accessed on January 23, 2016.

77. Bielecka A, Ambroziak AM, Skopiński P, et al. (2011): Zastosowanie kortykosteroidów w okulistyce. Kontaktologia i Optyka Okulistyczna 1: 7-10.

78. Langwińska-Wośko E, Bielecka A, Ambroziak AM, et al. (2011): Niesteroidowe leki przeciwzapalne w okulistyce. Okulistyka 3: 17-20.

79. Dursun Altınörs D, Suveren E, Karabay Ü, Aydın Akova Y (2010): Effects of human breast-milk on corneal epithelial healing in a in vitro model. AAO\&MEACO 2001, Chicago.

80. Enríquez-de-Salamanca A, Castellanos E, Stern ME, et al. (2010) Tear cytokine and chemokine analysis and clinical correlations in evaporative-type dry eye disease. Mol Vis 16 : 862-873.

81. Li S, Sack R, Vijmasi T, et al. (2008): Antibody protein array analysis of the tear film cytokines. Optom Vis Sci 85: 653660. 